\title{
NOTE \\ au sujet de divers essais de repeuplements en Brochets réalisés en Allemagne avant 1942 (1)
}

\author{
par M. René CHARPY
}

Conservateur des Eaux et Forêt.s

La nature fait généralement mieux les choses que nous-mèmes. Cepen. dant, en matière de pisciculture, les repenplement artificiels donnent parfois des résultats appréciables el encourageants. C'est le cas notamment des repeuplements ent Brochets réalisés dans l'Untersee, de rgo? et surtout de 1925 à 1942 .

Dans ce lac, où l'on capturait annuellement jusçu'à 42.500 kilogs de Brochets en 1895 , on n'en prenait plus qua 9.500 kilogs en 1910 , la régression du Brochet étật imputable, du moins en partie, aux masses d'eau polluées, déversées dans le lac, et aussi à la suppression de bon nombre de frayères naturelles, par suite des constructions croissantes en. treprises sur ses bords.

Ces constatations ont motivé l'effort considérable qui a été accompli tant par les Suis ses que par les Allemands pour la reconstitution du cheptel Brochet do l'Untersee.

Trois établissements ont contribué à son rẹpeuplement en Brochets, à savoir :

- En Suisse, l'établissement d'Ermatingen (dans le canton de Thurgau) clatant de 1897 et quí, de rq26 à i 942 , a déversé dans lé lac 67.471 .000 alevins de Brochets;

- En Allemagne, l'établissement de Radolfzell, créé en 1886, mais qui ne s est spécialisé dans l'ésociculture qu'à parlir de Igoo, et qui, de r926 à 1942 , a fourni à l'Untersee 33.885.00o alevins de Brochets ;

- Enfin, et surtout, en Allemagne encore, l'établissement de Reichenau, qui, créé en 1925 , a permis, de 1926 à 1942 , de déverser dans le lac 102.920,000 alevins de Brochets.

$\Lambda$ partir de ces trois établissements, l'Lntersec a donc reç, de I $_{2} 6$ à

(1) Cette note est rédigéc d'apres les deux articles suivants :

a) Oscar Roch, Inspecteur domanial du Service de la Pêche dans l'Untersee et le Rhin, La Pêche et l'Incubation du Brochet dans l'Untersee: - Allgemeine Fischerei-Zeilung, Janvier 1943, no 1, pp. 1 et 2.

b) Dr. Mast, Essais d'ćlevage de brochets. - Allgemeine Fischerei-Zeitung, 15 Mars $1939, \mathrm{n}^{\circ} 6, \mathrm{pl}, 89$ à 94. 
1942, 204.276.000 alevins de Brochets, soit près de 13 millions d'alevins de Brochets par an, durant i6 ans.

De la sorte, les captures de Brochets dans l'Untersee se sont accrues notablement pour atteindre déjà 25.500 Kilogs par ap en y 936 .

Tous les repeuplements dont il vient d'être rendu compte ont été effectués à l'aide de jeunes alevins de Brochets, encore pourvus, probablement, de leurs ventouses.

D'autres essais ont été réalisés ailleurs, mais sur une échelle beaucoup plus modeste, à l'aide non plus d'alevins, mais d'cufs frat̂chement fécondés.

Nous analysons ci-après les résultats obtenus :

En rg32, l'Association des pecheurs de l'arrondissement d'Augsbourg (au nord-ouest de Munich), a acheté un terrain de 2 hectares dans lequel 7 étangs ont été aménagés, à savoir : 3 étangs de 2.000 mètres carrés chacun (40 mètres sur 50 mètres), 4 de 150 mètres carrés chacun (40 mètres sur 4 mètres), et enfin I de 150 mètres carrés.

Expérience de 1933. - En 1933, seul l'un des grands étangs (de 2.000 mètres carrés) a élé utilisé pour le Brochet.

La chute de la rivière n'étant pas suffisante pour permeltre l'installation de bouteilles d'incubation, les oufs, fraîchement fécondés, ont été déversés directement dans l'étang, dont le fond était pourvu d'une végétation intacte. En dehors des œufs de Brochet rien n'a été mis dans l'étang.

Lors des marchés qui se tenaient à Augsbourg le vendredi, on choisit chez les pècheurs et marchands de poissons, des Brochets mûrs pour la fraye et l'on procèda immédiatement, comme pour une Truite, à la técondation artificielle.

Les pêcheurs étaient largement indemnisés pour la perte de poids subie par l'enlevement des ceufs.

La première fécondation eut lieu le $7 \mathrm{Avril}$. Deux autres furent pratiquées à ıo jours d'intervalle. La dernière eut donc lieu le 27 Avril, date à laquelle les alevins issus de la première fécondation commençaient déjà à

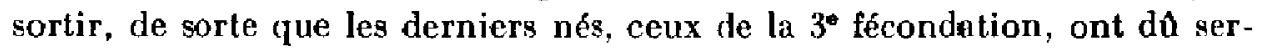
vir de nourriture aux autres.

Deux kilogs cinq cent d'œufs de Brochets, soit environ 1 i 6.000 cufs (I) ont ainsi été déversés dans cet étang de 2.000 mètres carrés.

A mi-Juin, il fut trouvé sur la digue, un jeune Brochet qui, manifestement, avait dú être saisi par un oiseau de proie : il mesurait déjà 3 a centimètres de longueur.

(1) En tablant sur 51.000 cufs au litre et sur 1.100 grammes par litre, chifire qui serait toutefois à vérifier. 
L'étang fut vidé le 23 Septembre 1933 . On y trouva 700 jeunes Brochets seulement. Par contre, le plus gros pesait 550 grammes et mesurait 42 centimètres de longueur. Quant aux longueurs des autres, elles s'étageaient entre 12 et 42 centimètres. Par acquit de conscience, un examen des écailles fut effectué et l'on put ainsi vérifier qu'il s'agissait bien là de types de un été. Il était d'ailleurs impossible que d'autres poissons pénètrent dans l'étang, par suite de l'existence d'un tamis sur la conduite.

Si donc, au cours de l'été, la croissance des brochetons fut considérable, le rendement en nombre fut extrêmement faible, même pas de l'ordre de 7 pour r.coo. Il y a lieu de noter toutefois que, comme dit plus haut, aucune nourriture n'ayant été mise à la disposition dẹs brochetons, ceux-ci ont dâ s'entredévorer, les derniers-nés devenant la proie des premiers.

Expérience de 1934. - En 1934 , trois des petits étangs (4 mètres sur 40 chacun) furent pourvus d'œufs de Brochets fraîchement fécondés, ceci les 23, 28 et 29 Mars pour les deux premiers, et le 13 Avril pour le trojsième (dans cet étang, les oufs, en petit nombre, provenaient de Brochets de I' $\Lambda$ mmersee, Brochets dont la fraye est plus tardive que celle des Brochets de rivière).

Comme en 1933, 2 kilogs 500 d'cuís furent utilisés.

L'étang dans lequel les ceufs avaient été dévetrsés en premier fut pêché le 13 Juin : on en retira 300 jeunes Brochets de 10 à 25 centimètres de longueur.

Le deuxième, pèché le 23 Juin, donna 2 ro brochetons des mêmes dimensions.

Quant au troisième, il ne donna que to jeunes Brochets.

Au total, le nombre de brochetons pêchés fut donc de 55o, résultat, dans un sens supérieur à celui obtenu lors de l'expérience de 1933 , puisque ces 550 brochetons furent obtenus dans 480 mètres carrés seulement, alors que les $7^{00}$ de l'année précédente l'avaient été dans 2.000 mètres carrés.

Toutefois, le rendement en nombre fut plus faible encore que celui de rg33 et n'atteint pas 4 pour r.ooo.

Expérience de 1935. - Cette année-là, des géniteurs Brochets furent capturés dans la riviēre la Schmutter, à 10 minutes au plus de l'établisset ment d'Augsbourg, où ils purent donc être amenés rapidement et sans heurts.

Six géniteurs furent placés à l'amont de l'un des grands étangs (de 2.000 mètres carrés). Il y avait là, grâce à la végétation, de belles frayères à brochets. Celles-ci furent isolées du reste de l'étang par un grillage. Or, dès le 20 Avril, on pouvait apercevoir des alevins de Brochets dans l'élang et, à la pêche réalisée le 5 Juin, on captura 400 brochetons un peu plus gros, dans l'ensemble, que ceux de 1934 .

On peut en conclure que des géniteurs-Brochets traités avec soin et 
n ayant pas subi des transports fatigants, peuvent êtra amenés jusqu'à la fraye, dans des étangs appropriés.

L'expérience toutefois - quant au nombre des brochetons obtenus fut en partie faussée du fait que, malgré la séparation, des géniteurs purent passer sous le grillage et, par conséquent, dévorer un certain nombre de brochetons.

Expérience de 1936. - Des essais analogues furent réalisés en rg36 mais, cette fois-ci, les génileurs furent achetés au marché.

Or, bien que ceux-ci aient été particulièrement choisis et ne présentaient aucunc blessure, un bon nombre ne frayèrent pas et presque tous périrent.

Les quelques ceufs obtenus des Brochets subsistants, furent placés sur des frayères artificielles, constituées par des branches de sapin posées sur des cages grillagées, celles-ci étant fixées, en faible courant, soit dans un ruisseau, soit à l'amont des étangs, soil sur les bords de ceux-ci. Or, tous les oufs, dont la plupart cependant devaient ètre fécondés, moisirent et ne donnèrent aucun alevin.

En somme, les Brochets qui, après leur capture font l'objet de transports et d'entrepôts divers (entrepờts après la peche, transports aux marchés, entrepôts à ces marchés dans des bacs souvent étroits, transports aux piscicultures, etc...) sont extrèmement fragiles, quelque robustes qu'ils puissent paraître. Les Brochets, fatigués par les voyages el la conscrvation, te sont plus bons à rien.

(Si, en 1933, des résultats satisfaisants furent obtenus à l'aide d'coufs prélêvés sur des Brochets achetés au marché, il faut tenir compte de ce que I'on a évité à ces Brochets un nouvel entrepôt, à savoir à la pisciculture; d'antre part, ils n'ont pas eu a fournir enx-memes l'eflort de la fraye).

Expérience de 1937. - Mêmes résultats négatifs en 1937 avec des Brochets paraissant sains mais ayant subi un assez long parcours; les poissons ont péri dans les huit premiers jours, sans être parvenus à frayer.

En outre, cette année-là, 2o.ooo alevins de Brochets, venant de Silésie, furent répartis le 24 Avril dans les quatre grands étangs.

En Juillet, on n'y retrouva que 7 oo brochetons, d'où un déchet considérable (le rendement fut toutefois de 35 pour 1.00o).

Doit-on, comme le voudnait le $D^{r}$ Mast, tirer de ces chiffres, des conclusions valables sur les déchels à intervenir lors du repeuplement des eanx libres. C'est discutable.

Si, en effet, dans les étangs de la pisciculture d' lugsbourgr, les brochetons n'ont pas rencontré autant d'ennemis qu'ils en auraient soufiert en eau libre, il faut tenir comple de ce que, dans ces étangs, aucune proie vivante n'a été mise à leur disposition.

En outre, il y á, pour les repeuplements, et suivant les cas, une densité optimum, qui serail à étudier. 
Expérience de 1938. - En I938, 20.000 alevins de Brochets, en provenance de la pisciculture de Lindau, furent déversés les ${ }^{\text {er }}$ et 14 Juin dans les grands étangs, ceux-ci étant, les uns, déjà pourłus de Carpes, les autres dépourvus.

Bien que ces alevins fussent arrivés en bon état, les résultats furent des plus médiocres, aussi bien dans lek étangrs à Carpes que sans Carpe; le rendement, en effet, ne fut que del l'ordre de 10 pour r.ooo. L'un des grands étangs donna 125 pelits Brochets; deux autres en donnèrent au total 80 , et, d'une façon générale, ces brochelons étaient plus petits que les années précédentes.

Au fail, cet insuccès n'est pas seulement imputable au transport fatigant de Lindau à Augshourg (i5 x kilomètres), mais aussi aux destructions faites par les Carpes elles-mèmes, et, enfin, aux froids survenus en Avril et Mai, froids qui ont entravé le développement normal de la nourriture susceptible d'être utilisée par leg Brochets.

Il faut noter également que dans les étangs de la pisciculture d'Augsbourg, du moins cette année-là, les larves de "Gelbrandkäfer " (sorles de Dytiques), pullulaient à tel point quaux dires mèmes du I ${ }^{r}$ Mast, il y an avait plus que de brochetons. Il fallait, écrit le J $^{r}$ Mast, s'efforcer de trier les petils Brochets dans le tumulte effarant des chabots et des larves de coléoptères.

Les chiffres des rendements obtenus par te $D^{r}$ Mast au cours des divers essais de repeuplement tentés par lui de ig33 à 1938 , ne sauraient avoiı évidemment, qu'une valeur relative, d'une part, parce que ces essais n'ont jamais fail l'objet que d'une seule expérience dans chaque cas particulier ; d'autre part, parce que ces essais $n$ 'ont pas toujours été réalisés dans des conditions particulièrement favorables : manque de nourriture, surabondance des insectes nuisibles, densité du repeuplement fixée à priori.

Les conclusions à en tirer, par la comparaison de ces divers rendements, ne peuvent donce elles-mêmes; n'être que relntives et sujettes à révision.

Néanmoins, les expériences relatées ci-avant nous ont paru présenter suffisamment d'intérêt pour faire l'objet de l'analyse critique à laquelle nous avons procédé.

Il convient maintenant, sous les réserves indiquées plus haut, de'tenter de tircr de ces expériences, des conclusions plus générales c'est ce à quoi nous nous attacherons ci-après :

$I^{\circ}$ De toute façon - du moins dans l'état actuel de nos connaissances les déchets constatés lors des repeuplements effectués avec des alevins de Brochets, sont extrêmement importants. C'est ainsi que le rendement obtenu en ${ }^{3} 9^{3} 7$ par le $D^{r}$ Mast n'a été que de 35 pour r.ooo.

Certes, en 1938, ce rendement n'a été que de so pour 1.000, mais cette année-là, les alevins ont été placés dans des bassins restreints, dont la 
plupart étaient déjà occupés par des Carpes et qui étaient, en outre, infestés par des Chabots et des larves de Dytiques.

Ori peut donc approximativement tabler, dans le cas le moins défavorable des expériences du $\mathrm{D}^{r}$ Mast, sur un rendement, avec des alevins, de l'ordre de 35 pour 1.00o. Ceci motive, semble-t-il, l'importance de l'effort qu'il a fallu accomplir pour la reconstitution du cheptal-Brochet de l.Untersee ;

$2^{\circ}$ Mais nous ne saurions admettre, avec le $D^{r}$ Mast que "l'empoissonnement avec des alevins a, par endroit, totalement échoué ", car si le déchet sur les alevins est très important, il l'est beaucoup plus cncore dans le cas de lemploi des ceufs fraîchement fécondés, ce dont le $\mathrm{D}^{\mathbf{r}}$ Mast aurait pu se rendre compte, en cherchant à déterminer, au moins approximativement, - ce qu'il n'a point fait, - le nombre d'ceufs compris dans les $2 \mathrm{k}$. 5̆oo d'cufs utilisés par lui tant en 1933 qu'en 1934.

En effet, les rendements oblenus en 1933 et 1934 avec des ceufs fraichements fécondés, ont été respectivement de l'ordre de 7 pour 1.000 et de 4 "pour I.000 seulement.

Nous ne saurions donc partager l'optimisme du Dr Mast lorsqu'il declare que " dans le domaine de l'emploi des oufs de Brochets fécondés, il a obtenu les meilleurs résultats !" $\mathrm{Si}$ " pour le développement de l'élevage du Brochet, des établissements pisicoles et des appareils d'incubation ne sont pas indispensables " et si "cet élevage acquiert, de ce fait, une base beaucoup plus étendue que celle admise jusqu'à préscnt ", nous ne considérons pas la chose comme "un fait capital ", étant donné les résultats vraiment très faibles, obtenus jusqu'alors dans I'emploi des œufs de Brochet, fraîchement fécondés.

Ceci ne veut pas dire, d'ailleurs, que ces expériences ne soient pas extrêmement intéressantes et qu'il n'y ait pas lieu de poursuivre des recherches systématiques dans ce sens, étant donné la simplicité du système et le peu de frais qu'entraîne sa mise en application ;

$3^{\circ}$ Nous pensons que si les repeuplements avaient été réalisés non plus à l'aide d'oufs fraîchement fécondés, mais bien d'ceufs déjà embryonnés (ce qui implique, il est vnai, l'usage de bouteilles d'incubation), les résultats auraient peut être été meilleurs.

Si le rendement, par emploi d'œufs embryonnés, peut atteindre (et nous sommes portés à le croire) celui à escompter en utilisant des alevins, il est bien certain que I'utilisation d'œufs embryonnés - dont le transport est beaucoup plus facile que celui des alevins - est à enviseger ;

$4^{\circ}$ ll convient, comme l'a montré le $\mathrm{D}^{\mathrm{r}}$ Mast, que les géniteurs-Brochets n'aient pas à faire l'objet de transports fatigants, juste avant l'époque de la fraye. Les faits relatés, à ce sujet, par le $D^{r}$ Mast, corroborent ceux que nous avons pu constater nous-mêmes, notamment à l'établissement domanial de pisciculture de Neuville-en-Sainte-Gemme, dans la Marne : 
en 1939 , par exemple, nous avons acheté, pour les besoins de cette pisciculture, des géniteurs-Brochets qui, capturés dans des étangs de la Somme, avaient été entreposés provisoirement dans les bassins de la Station d'Hydrobiologie du Paraclet. De là, en prenant toutes les précautions d'usage, nous les avons transportés à la piscicultura de Neuville où, quatre heures après, ils arrivaient, en bon état, semblait-il.

Or, ces Brochets, au nombre d'une quinzaine environ, placés cependant dans des bassins relativement vastes et bien alimentés en eau, sont tous morts les uns après les autres. Seule, une femelle a pu frayer et être fécondée en temps opportun.

Mais, au fait, si los longs transports indisposent les géniteurs-Brochets et si la stabulation leur est néfaste, nous sommes persuadés que la température de l'eau joue un rôle considérable. En particulier, si celte température est inférieure à celle de l'eau d'où proviennent les géniteurs (et c était le cas pour Neuville), on peut avoir les plus amers déboires.

Tenu compte de ces diverses considérations, nous avons pris à Neuville, dès après guerre, toutes dispositions en vue d'avoir sur place le cheptelgéniteurs nécessaire : les Brochets furent placés dans les étangs mêmes de la pisciculture et ces étangs furent paurvus de blanchaille susceptible de leur servir de nourriture. Les manutentions furent ainsi réduites au minimum, la capture des géniteurs put être réaliséa peu de temps avant l'époque de la fraye et les Brochets furent toujours placés dans une eau dont la température restait à peu près constante ; de la sorte, la fécondation a pu donnęr des résultats satisfaisants.

Aussi nous rallions-nous entièrement à l'avis émis sur ce point par le $D^{r}$ Mast lorsqu'il déclare qu'en matière d'ésociculture " la centralisation serait une utopie et ne pourrait mener qu'à l'insuccès ". Il faut, en effet, que les établissements d'incubation d'œufs de Brochets, quitle à ce que l'on en ait plusieurs, soient situés à proximité des centres de capture des géniteurs ;

$.5^{\circ} \mathrm{Si} \mathrm{l}^{\prime}$ ésociculture est assez délicate, elle n'en est pas moins extrêmement attrayante et intéressante, ne serait-ce que par la qualité des produits obtenus et leurs dimensions.

En Avril 1939 , exactement le $\mathbf{1}^{\text {ar }}$ Avril 1939 , nous avons pu obtenir, à Neuville, par la fécondation artificielle, un millier d'alovins de Brochets que, par petits paquets de 2 ou 3 , nous avons répartis tous les $1 \mathrm{~m}$. 5o environ, le long des berges de l'étang inférieur, étang qu'à dessein, nous avions bien pourvu de blanchaille (Gardons, Tanches, Carpes).

Durant les hostilités, ces étangs ayant été vidés et pillés, les alevins ont da gagner la rivière voisine, la Semoigne. Fort heureusement, des trous d'obus existant dans l'étang inférieur, nous avons pu retrouver là, so Brochets qui n'avaient pu en sortir. Mesurés le ${ }^{\text {er }}{ }^{\text {Avril }}$ ig4r, donc jour pour jour, 2 ans après leur naissance, ils mesuraient tous, entre 50 et 60 centi- 
mètres, dimensions déjà fort respectables, et qui, probablement, l'auraient été davantage encore, si les étangs avaient été laissés entièrement en eau ;

$6^{\circ}$ Le Brochet est un poisson carnassier, très vorace. Aussi le détruisaiton autrefois. En fait, ce qu'il mange, ce n'est jamais que de la blanchaille de faible valeur commerciale, qu'il transforme en une chair très estimée, atteignant en prix, celle de la Truite. D'autre part, depuis l'invention du lancer léger, les pècheurs capturent, dans nos cours d'eau, beaucoup plus de Brochets que jadis. Enfin, nous importions chaque anné, ne serait-ce que pour l'industrie de la quenelle, un assez fort contingent de Brochets.

Pour toutes ces raisons, il convient, en France, de développer les repeuplements en Brochets.

Aussi émettons-nous le vou que des recherches méthodiques soient poursuivies en vue de déterminer les meilleures conditions dans lesquelles repeuplements doivent être réalisés. 